\title{
APRECIACIONES CRÍTICAS A LOS PROGRAMAS DE CONTROL Y ELIMINACIÓN DE LA LEPRA EN EL PERÚ Y SUS CONSECUENCIAS PARA EL PERÚ Y AMÉRICA
}

\author{
CRITICAL APPRAISAL ABOUT CONTROL PROGRAMS AND ELIMINATION OF LEPROSY \\ IN PERU, AND ITS CONSEQUENCES FOR PERU AND AMERICA
}

Zuño Burstein ${ }^{1, a, b, c, d}$

Instituto Nacional de Salud. Lima, Perú.

Médico dermatólogo tropicalista; ${ }^{b}$ profesor emérito de la Universidad Nacional Mayor de San Marcos; ‘ académico honorario de la Academia Nacional de

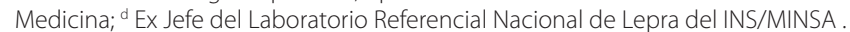

* Tema presentado en el curso: Hanseniasis de la XXX RADLA 2012. Sao Paulo Brasil, mayo 2012

Recibido: 12-05-14; Aprobado: 11-06-14

\section{RESUMEN}

Se presenta un análisis crítico de las medidas de control sanitario que, históricamente, se llevaron a cabo en el Perú hasta llegar a la situación actual, que sitúa al Perú en la fase oficialmente considerada de "eliminación" de la lepra como problema de salud pública. Se discute, además, su validación, señalándose que el descuido de la vigilancia sanitaria, la desarticulación de los organismos de control especializado, el olvido de parte del personal médico sanitario de su diagnóstico precoz, la presencia de casos lepromatosos bacilíferos no diagnosticados en la capital de la república y la indudable prevalencia escondida, permiten concluir que existe el peligro para el país y la región que se reproduzca una recrudescencia de la lepra, si no se toman las medidas sanitarias pertinentes y oportunas.

Palabras clave: Lepra; Prevención y control; Políticas públicas de salud; Perú (fuente: DeCS BIREME).

\begin{abstract}
A critical analysis of health control measures that historically took place in Peru to the present which has led Peru to officially consider leprosy as an "eliminated" public health problem. We will also discuss the validity of the status given the neglect of health surveillance, disbanded specialized control entities, health medical staff forgetting to account for leprosy in early stages, the presence of undiagnosed smear-positive leprosy in Lima and the undeniable hidden prevalence, suggest that there is a danger to the country and the region that a re-emergence of leprosy will occur, if relevant and appropriate sanitary measures are not taken.
\end{abstract}

Key words: Leprosy; Prevention and control; Health public policy; Peru (source: MeSH NLM).

\section{INTRODUCCIÓN}

Por homologación con el objetivo trazado y logrado en mayo de 1980 por el sanitarismo mundial de erradicar una de las plagas más mortíferas y temibles que azotó por milenios a la humanidad, que fue la viruela, la Organización Mundial de la Salud se trazó el objetivo de hacer lo mismo con la lepra ${ }^{(1)}$.

La viruela fue una enfermedad, producida por un Poxvirus de gran patogenicidad y virulencia, adaptado a la especie humana, altamente transmisible, que infectaba sin distinción de edad, raza, sexo ni condición social, produciendo alta letalidad, pero, que, afortunadamente, podía lograrse resistencia del huésped a la infección viral mediante manejo inmunológico y se consiguió, a través de un programa de vacunación universal, obligatorio y compulsivo, que la enfermedad fuera erradicada mundialmente.

Con este éxito, se pensó que la lepra, enfermedad producida por una bacteria que, igualmente, da lugar a una enfermedad exclusiva de la especie humana, podría ser manejada con similares medidas. Desafortunadamente, esta enfermedad ofrece fundamentales diferencias con la viruela.

El Mycobacterium leprae, agente causal de la lepra o enfermedad de Hansen, requiere condiciones especiales para manifestar su patogenicidad. Existen factores

Citar como: Burstein Z. Apreciaciones críticas a los programas de control y eliminación de la lepra en el Perú y sus consecuencias para el Perú y América. Rev Peru Med Exp Salud Publica. 2014;31(2):336-42. 
determinantes en la progresión y presentación de la enfermedad, entre ellos los condicionamientos inmunogenéticos de receptividad individual y, predominantemente, las alteraciones inmunorreactivas determinadas por factores ambientales, los cuales deprimen los mecanismos de defensa a la invasión bacteriana, especialmente en grupos poblacionales con déficits nutricionales, en condiciones de hacinamiento, así como un conjunto de factores dependientes de la pobreza, propios de países subdesarrollados ${ }^{(2)}$.

La pobre respuesta a los agentes inmunizantes hace poco efectiva la posibilidad de utilizar recursos inmunógenos para el control de la lepra, pues en esta enfermedad existen evidentes defectos parciales y totales de la inmunidad celular con trastorno de la secreción de citoquinas, mientras que la inmunidad inmediata permanece normal ${ }^{(3)}$. La secreción de diversas citoquinas por efecto del $M$. leprae puede trastornar la función inmunitaria, lo que ocasiona a esta función defectos importantes, sin existir explicación satisfactoria de las causas de estos defectos.

La Asamblea Mundial de la Salud, realizada el año 1990, decretó, como parte de su propuesta para eliminar la lepra como problema de salud pública para el año 2000 , reducir la tasa de prevalencia a cifras inferiores a 1 por 10 mil habitantes, niveles en los que el potencial de transmisión es muy limitado, ello no significa la interrupción de la transmisión ni la erradicación de la enfermedad ${ }^{(4)}$. Sin embargo, esta propuesta tuvo un importante significado, considerando que la lepra fue, durante muchos años, una enfermedad donde las acciones para su control se realizaban de forma muy limitada y sin muchas esperanzas ${ }^{(5,6)}$.

Esta modificación radical en la estrategia fue posible al disponer de la poliquimioterapia (PQT), una tecnología de tratamiento apropiada y robusta, que ha demostrado ser de alta eficacia, efectividad y factibilidad, recomendada por el "Grupo de Estudio de la OMS sobre

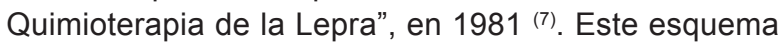
de tratamiento combina dos o tres medicamentos, de acuerdo con la forma clínica de la enfermedad, con lo que se evita el desarrollo de cepas resistentes de $M$. leprae y se logra eliminar al enfermo como fuente de infección desde las primeras dosis que se aplican. El tratamiento permitió afirmar, por primera vez, con una base científica firme, que la lepra se cura y que las acciones para su control pueden ser gerenciadas y ejecutadas por los servicios generales de salud, como la mayoría de las enfermedades transmisibles. Por otra parte, el reforzamiento de los programas y el incremento de las acciones que se han producido a partir de la PQT, han prevenido las incapacidades en más de un millón de pacientes, mediante el diagnóstico precoz y el tratamiento oportuno.
Un aspecto de gran importancia es que la lepra dejó de ser vista como "un problema de la piel" y comenzó a ser atendida como una enfermedad transmisible. Sin embargo, un estudio realizado en Perú sobre conocimientos, actitudes y creencias del personal de salud acerca de la enfermedad de Hansen (8), concluyó que el nivel de conocimientos resulta ser limitado entre los profesionales, especialmente los médicos. Persisten conceptos errados sobre la enfermedad que son transmitidos entre la comunidad y el personal de salud, que se expresan en percepciones y actitudes frente al paciente; en dicho estudio se señala que los currículos de formación y programas de entrenamiento de los futuros recursos humanos de la salud se encuentran desvinculados de la realidad sanitaria, lo que determina un escaso nivel de información acerca de las enfermedades más prevalentes en el país, como es el caso de la lepra, ahondando cada vez más las dificultades existentes para ejercer un adecuado control de la endemia.

\section{ANÁLISIS CRÍTICO DE LAS PRIMERAS MEDIDAS DE CONTROL DE LA LEPRA EN EL PERÚ}

En el Perú, los antecedentes inmediatos de la constitución de un organismo científicamente preparado y técnicamente estructurado que permitiera una acción de control a nivel nacional ${ }^{(9)}$, los tenemos en la rápida progresión de la endemia leprosa loretana (en la selva peruana), generada a raíz de la "fiebre del caucho" que, en el último decenio del siglo antepasado, determinó la migración de gran número de braceros loretanos hacia el Brasil.

A comienzos del siglo pasado, al ser desplazado el caucho amazónico por las plantaciones holandesas de Indonesia, se inició el retorno de esos grupos humanos que habían estado residiendo cerca de 20 años en zonas endémicas de lepra, en los estados de Maranhao, Pará y Amazonas, a partir de los cuales dos corrientes de infección penetraron en el suelo loretano: una que siguió el curso del río Amazonas aguas arriba, se diseminó hasta los orígenes del río Ucayali medio y bajo; la otra corriente infecciosa, haciendo estación secundaria en el territorio del Acre, alcanzó la hoya ucayalina a través de los ríos Yurúa y Purús, aprovechando el comercio de jebe fino establecido entre estos dos territorios.

En 1905 se construye el primer lazareto en la ciudad de Iquitos; en 1906 se ordena la construcción de un asilo para leprosos en la isla Padre, frente a lquitos; en 1917 se habilitó en la misma ciudad un segundo lazareto. Pero es en 1926 cuando se inicia la actividad antileprosa estatal, al ponerse en servicio, el 15 de mayo de ese año, una leprosería en San Pablo, en el rio Amazonas, en la frontera con Brasil. Mientras tanto, Hugo Pesce, 
en Andahuaylas, detecta los primeros casos de lepra andina y crea, el año 1937, el servicio antileproso de Apurímac. El año 1941 se reconstruye el Asilo de San Pablo como colonia agrícola, y el 1 de enero de 1944, Hugo Pesce crea la Campaña Nacional Antileprosa, como organismo sanitario encargado oficialmente de la lucha antileprosa a nivel nacional. Nace, así, en torno a ese maestro, la escuela leprológica peruana y, el mismo año, se constituye el Servicio Antileproso del Nororiente. El rasgo estructural de la campaña antileprosa, que en 1954 pasa a denominarse "Servicio Nacional Antileproso", estaba dado por ser un organismo unitario con una jefatura denominada Departamento de Lepra y diversos servicios periféricos. Esta organización metódicamente planeada, puesta en marcha, permitió, en un corto plazo, hacer el diagnóstico de la realidad leprológica peruana y obtener un beneficio efectivo para los pacientes y el país ${ }^{(10)}$.

Desgraciadamente, el 14 de enero de 1963, el Departamento de Lepra, transformado previamente en División de Lepra, fue disuelto por el gobierno de aquel entonces y su jefe, el doctor Hugo Pesce, destituido por razones incalificables de motivación política. La estructura tan meticulosamente montada se desarticula, sus diferentes elementos constitutivos son incorporados a otros organismos y, desde el año 1965, los niveles periféricos son integrados con los otros servicios de salud en cada zona del país. El programa de control de la lepra, ya no individualizado, no priorizado y siguiendo la optimista corriente universalmente aceptada de que no es un problema sanitario que requiera tratamiento especial ni diferenciado, termina siendo incluido, en desventaja, con otros problemas, minimizado y olvidado a nivel de los planes operativos a cargo del Estado ${ }^{(11)}$.

La ejecución de las acciones de salud relacionadas con la enfermedad de Hansen, de acuerdo con la política vigente, pasaron a cargo de las áreas hospitalarias, órganos desconcentrados regionales, enmarcados en una tónica de descentralización político administrativa del país y de las direcciones regionales de salud, directamente dependientes de la Dirección Superior del Ministerio de Salud. Los recortes presupuestales para el sector salud y la crisis económico - financiera por la que atravesaba el país imposibilitaban a los organismos ejecutores otorgar la más mínima atención a las normas establecidas por la unidad técnico normativa de nivel central. El diagnóstico especializado de laboratorio, la preparación de lepromina, la realización de investigaciones especiales y la capacitación del personal profesional y técnico estaba en manos del Departamento de Lepra y Micología Médica, ubicado en la estructura organizativa de los Institutos Nacionales de Salud, organismo descentralizado del Ministerio de Salud.
En este, como en los otros casos, lo precipitado de las medidas reorganizativas, la equivocada actitud a nivel estatal de minimizar el problema, basada en un falso optimismo de que este se resolvería con la becegización e integración a los programas de control de otras enfermedades transmisibles y a su baja contagiosidad, no permitieron el establecimiento de una red orgánica de coordinación de actividades y un sistema adecuado de trabajo a nivel nacional, utilizando racionalmente los recursos humanos e infraestructura existentes. A esto se sumó la competitividad agresiva de personas interesadas en utilizar, por cualquier medio, recursos financieros nacionales y extranjeros otorgados a nombre de la lucha antileprosa, algunos realmente aplicables a aspectos relacionados con la investigación de la afección, pero, la mayor parte usados para otros fines, algunos quizás loables, pero otros francamente ilícitos.

\section{EVOLUCIÓN DE LOS PROGRAMAS DE CONTROL Y ELIMINACIÓN DE LA LEPRA EN EL PERÚ DESPUES DE LA DISOLUCIÓN DEL SERVICIO NACIONAL ANTILEPROSO}

La lepra en América no existía antes de la llegada de los conquistadores europeos. Los españoles trajeron la lepra a América Central, América del Sur y, en Norteamérica, a México y parte de los Estados Unidos. El primer lazareto se fundó en 1520 en Santo Domingo $y$, posteriormente, se establecieron en toda la América colonial. En Brasil, los portugueses introdujeron la enfermedad desde 1496; los grandes contingentes de esclavos africanos fueron un factor muy importante en la América portuguesa, Caribe y América Central. En Norteamérica, además de los focos traídos por los españoles, se sumaron los traídos por los franceses, noruegos e inmigrantes chinos, principalmente.

La historia de la lepra en el Perú ha sido exhaustivamente estudiada por el Dr. Hugo Pesce y publicada en su tesis de doctorado el año 1961, con el nombre de "La epidemiología de la lepra en el Perú" (9). En este monumental trabajo, que debe servir de valiosísima fuente informativa para todo médico y sanitarista, se afirma que la lepra en el Perú se ha desarrollado de manera independiente en las tres grandes regiones del país, denominadas costa, sierra y Amazonía.

El Servicio Nacional Antileproso, creado por Hugo Pesce, con su Jefatura, el Departamento de Lepra, fue disuelto el 14 de enero de 1963, sus diferentes estructuras constitutivas fueron pasadas a otros organismos $y$, desde 1965, los niveles periféricos fueron integrados otros servicios de salud de cada zona del país. 
La Dirección de Normas y Supervisión de Salud del Ministerio de Salud Pública y Asistencia Social, a través de la Asesoría de Tuberculosis, publica y difunde, en julio de 1967, el documento denominado "Normas y Procedimientos para los Programas de Control de Tuberculosis y Lepra"; dentro de las "Normas para el Control de la Lepra" se estableció que: Cada área de salud deberá elaborar sus programas de control de enfermedades trasmisibles, en que estarán incluidas las actividades antileprosas de su jurisdicción, cuyas comunicaciones deberán hacerse por los canales regulares correspondientes, con información directa a la Asesoría de Tuberculosis y Lepra, y que: Desde el nivel central debe ejercerse una supervisión periódica de todos los servicios en los que se apoya. Este documento incluye un "Manual de Procedimientos para el Programa de Control de Lepra".

En 1979, la Unidad de Lepra de la Dirección de Erradicación y Control de Enfermedades Transmisibles del Ministerio de Salud, publica el "Programa de Control de la Enfermedad de Hansen 1979", en el que señala la doctrina y política, objetivos y metas, y estructura organizativa a nivel nacional, asimismo, establece las "Normas, procedimientos y funciones para la ejecución del Programa de Control de la Hanseniasis a nivel de área hospitalaria". El 2 de enero de 1984, por Resolución Ministerial 0002-84-SA/AVM, del Ministerio de Salud, se aprueba el "Manual y procedimientos de la enfermedad de Hansen", encargándose a la Dirección General de Atención a las Personas, a través de la Dirección de Enfermedades Transmisibles, su difusión y aplicación.

En enero de 1988, por Decreto Supremo 003-88-SA y Resolución Ministerial 020-88-SA/DM, se crea y aprueba el "Programa Nacional de Control de la Hanseniasis", desligado del Programa de Control de Tuberculosis, como integrante de los programas especiales de salud, a cargo de una dirección general y, el 20 de octubre de 1988, por Resolución Ministerial 384-88-SA/DM, se aprueban las "Normas y procedimientos para el control de la hanseniasis en el Perú", de aplicación obligatoria en todo el territorio nacional.

En 1992, se publica la primera edición de la "Doctrina, normas y procedimientos para el control y eliminación de la lepra en el Perú", aprobado por R.M. 0160-92-SA/DM, la que fue sometida a sucesivas revisiones y actualizaciones. Actualmente, promulgada por Resolución Ministerial, se encuentra en vigencia desde el 27 de diciembre de 2005, la última "Norma Técnica de Salud para la eliminación de la lepra”, elaborada con colaboración interinstitucional por la Estrategia Sanitaria Nacional de Prevención y Control de la Tuberculosis Componente Lepra, dependiente de la Dirección General de Salud de las Personas del Ministerio de Salud del Perú (12).

\section{CONVENIO ENTRE EL MINISTERIO DE SALUD Y LA UNIVERSIDAD MAYOR DE SAN MARCOS}

En 1975, mediante un convenio interinstitucional, renovado sucesivamente, con vigencia hasta la fecha, se reubica el Departamento de Lepra y Micología Médica de los Institutos Nacionales de Salud en los ambientes físicos del Instituto de Medicina Tropical "Daniel A. Carrión" de la Universidad Nacional Mayor de San Marcos (UNMSM) y se incorporó funcionalmente a su estructura organizativa como Servicio de Dermatología Sanitaria del Instituto de Medicina Tropical "Daniel A. Carrión” de la UNMSM.

En 1985, el Departamento de Lepra y Micología Médica de los Institutos Nacionales de Salud, que había cambiado de denominación por Servicio de Hanseniasis y Dermatología Sanitaria, conservando todas sus funciones, desaparece del organigrama del Instituto Nacional de Salud y es incorporado al organismo central del Ministerio de Salud como Laboratorio Nacional de Referencia de Hanseniasis del Programa Nacional de Control de Tuberculosis y Hanseniasis de la Dirección de Epidemiología de la Dirección General de Servicios de Salud.

El año 2005 se dispone el retorno del Laboratorio Nacional de Referencia de Lepra a su lugar de origen, el INS, pero no se formalizó esta reincorporación que quedó como una estructura virtual, cumpliendo con sus responsabilidades funcionales pero físicamente en el Instituto de Medicina Tropical de la UNMSM incorporado en su Servicio de Dermatología Sanitaria y en su Laboratorio de Anatomía Patológica, haciendo uso para este efecto del convenio interinstitucional. Este laboratorio se encuentra actualmente en latencia, a la espera de las decisiones que adopten los funcionarios con responsabilidad en su funcionamiento y estructura.

Un buen ejemplo de la colaboración de algunas instituciones privadas, como fue el caso de la ONG ANESVAD - Servicio de Medicina PRO-VIDA, Io tenemos en el desarrollo de un curso de capacitación al personal de salud de Tarapoto en la selva peruana como parte del proyecto "Búsqueda activa, diagnóstico y tratamiento de pacientes con lepra en Amazonas y San Martin, para contribuir a la eliminación de la lepra en el Perú".

\section{SITUACION ACTUAL DE LA LEPRA EN EL PERÚ}

La lepra en el Perú muestra oficialmente una prevalencia menor al 1 × 10000 habitantes, considerándose, por lo tanto, que se ha llegado a la fase de la eliminación 


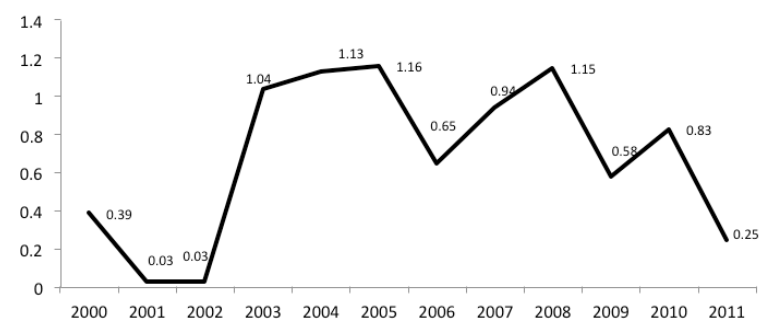

Figura 1. Tasa de prevalencia de lepra por 10000 habitantes en Perú, 2000-2011

Fuente: ESN PCT y lepra 2001-2011

de la enfermedad; pero esta afirmación merece ser cuestionada por los siguientes motivos.

El comportamiento epidemiológico de la lepra se circunscribe a zonas endémicas de la Amazonía peruana; se podía afirmar hasta hace algunos años que constituía un problema de salud pública regional, como lo fuera Ucayali (13) con una prevalencia mayor de 1 × 10000 habitantes y áreas con prevalencias menores de 1 x 10000 habitantes como Loreto, San Martín, Amazonas y Huánuco; sin embargo, en algunos departamentos de la región andina, como Cajamarca, provincia de Jaén, se han reportado cinco casos en los últimos cinco años. En Ayacucho, que nunca tuvo casos, se han reportado recientemente dos pacientes con evolución entre 6 y 20 años de la enfermedad, con el agravante, de que acudieron varias veces a los establecimientos de salud de su región de origen sin ser diagnosticados.

Otro aspecto a tener en cuenta es el abandono de la búsqueda activa entre los contactos intra y extradomiciliarios de los casos detectados, se puede decir que todos estos factores son los que determinan, como consecuencia, la existencia de una prevalencia oculta de la lepra en las zonas endémicas del Perú que impiden su eliminación. La información oficial disponible del Ministerio de Salud del Perú de la Estrategia Sani- taria Nacional de Control y Prevención de la Tuberculosis Componente Lepra, se limita a un "Informe Gestión ESN-PCT-Componente Lepra" hasta el año 2006 y sus cifras no serían representativas de la real endemia de la enfermedad. Para la presente comunicación se ha solicitado al Ministerio información actualizada, la cual se muestra gráficamente, según la tasa de prevalencia de la lepra y su distribución en regiones del Perú durante los años 2001-2011 (Figura 1, Tabla 1).

\section{CASOS CLÍNICOS CON DIAGNOSTICO TARDIIO}

Se presentan dos casos de Lepra multibacilar florida diagnosticados en Lima, procedentes de regiones endémicas de la región selvática del Perú que se encontraban sin diagnóstico, a pesar de su largo tiempo de evolución y haber tenido atención médica.

Un primer caso (Figura 2) se trata de una adolescente procedente de Loreto con residencia en Lima y tiempo de enfermedad manifestada desde hace un año; se desempeña como trabajadora del hogar, y tiene una numerosa familia con la que vive en condiciones de hacinamiento, manifiesta haber sido atendida médicamente por acné nódulo quístico. A solicitud de un servicio hospitalario, y con colaboración nuestra, se hizo el diagnóstico y se inició el tratamiento de inmediato, proporcionado por la Estrategia Sanitaria de Prevención y Control de Tuberculosis - Componente Lepra. Pese a las indicaciones no se ha hecho el control y seguimiento de contactos. Dos meses después de terminado el tratamiento ambulatorio, y ya sin control hospitalario, regresa con lesiones activas, reiniciándose la terapia.

El segundo caso (Figura 3), visto en Lima en octubre del 2009 , es un adulto mayor, agricultor, procedente de la provincia de Huanta en el departamento de Ayacucho, con un tiempo referido de tres años de evolución, sin diagnóstico, a pesar de haber sido visto médicamente

Tabla 1. Casos de lepra reportados por regiones del Perú, 2000-2011

\begin{tabular}{|c|c|c|c|c|c|c|c|c|c|c|c|c|}
\hline & 2000 & 2001 & 2002 & 2003 & 2004 & 2005 & 2006 & 2007 & 2008 & 2009 & 2010 & 2011 \\
\hline Ucayali & 14 & 21 & 26 & 25 & 10 & 8 & 13 & 22 & 28 & 9 & 14 & 18 \\
\hline Loreto & 36 & 11 & 35 & 34 & 6 & 2 & 19 & 12 & 7 & 15 & 22 & 7 \\
\hline Amazonas & 0 & 5 & 1 & 1 & 7 & 9 & 10 & 1 & 2 & 2 & 1 & 0 \\
\hline Huánuco & 1 & 1 & 0 & 0 & 1 & 2 & 1 & 0 & 0 & 1 & 1 & 2 \\
\hline San Martin & 0 & 0 & 0 & 0 & 5 & 5 & 0 & 1 & 0 & 0 & 9 & 7 \\
\hline Ayacucho & & & & & & & & & & 2 & 0 & 0 \\
\hline La Libertad & & & & & & & & & & & & 1 \\
\hline Lima Este & & & & & & & & & & & & 1 \\
\hline Total & 51 & 38 & 62 & 60 & 29 & 26 & 43 & 36 & 37 & 29 & 47 & 36 \\
\hline
\end{tabular}

Fuente: ESN PCT y lepra 2001-2011 

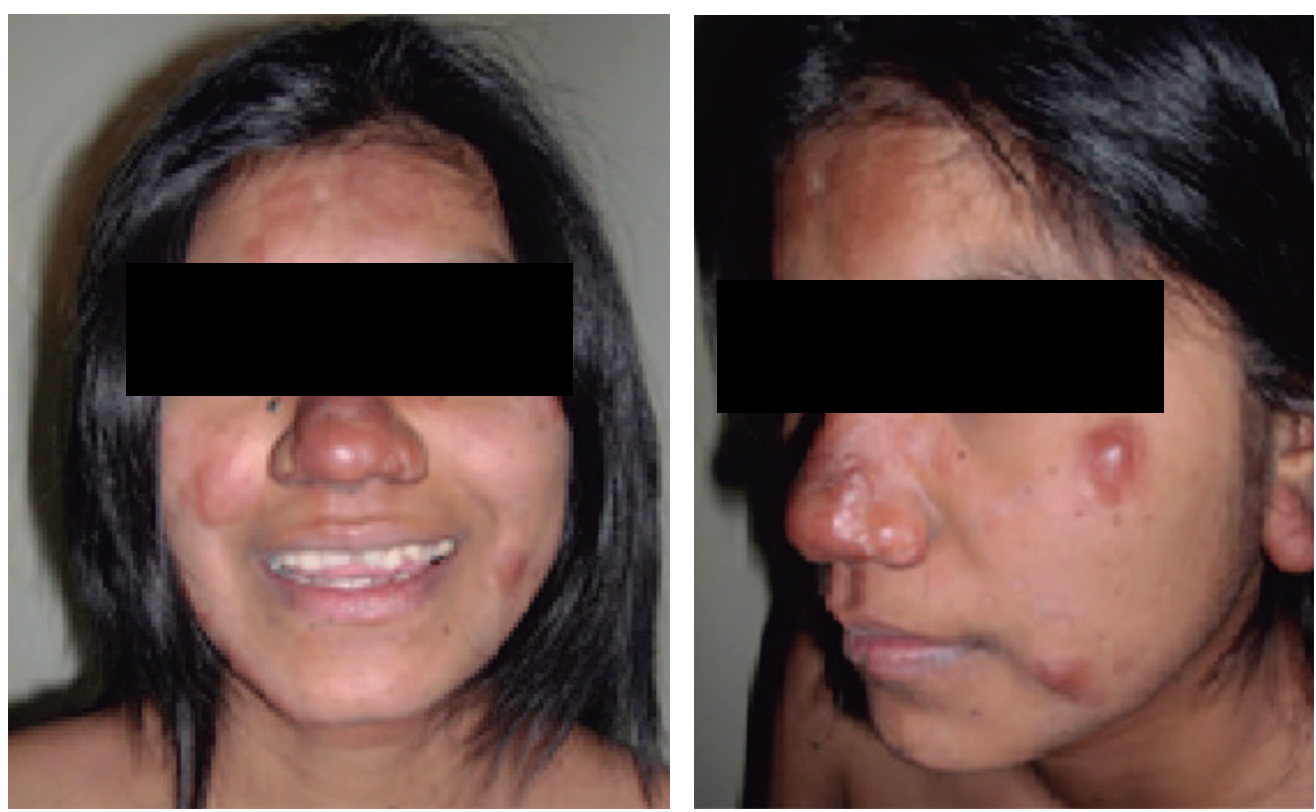

Figura 2.

Paciente con lepra lepromatosa diagnosticada en Lima; adolescente de 17 años de edad, procedente de Iquitos, Loreto, residente 1 año en Lima con el diagnóstico erróneo de acné nodular en su lugar de origen. Remitido por una Institución oncológica se hace el diagnóstico y se inicia el tratamiento PQT, de acuerdo con el esquema establecido, retornando a su ciudad para continuar el control.

\section{COMENTARIOS FINALES}

El Perú, de acuerdo con la información oficial, tiene una prevalencia de lepra a nivel nacional menor al 1 x 10000 habitantes, por lo que se le ubica entre los países que han logrado la eliminación de la enfermedad, de acuerdo con los criterios establecidos por la OMS. Pero esta información no es totalmente válida para los efectos de esta ubicación optimista, ya que la lepra en el Perú ha sido siempre una endemia regional con tasas que sobrepasaban estos índices en ciertas regiones endémicas en la región amazónica; aunque, oficialmente, se encuentran en la actualidad, por debajo del riesgo epidemiológico establecido, lamentablemente ofrecen una prevalencia oculta con la presencia de casos no detectados en ciudades como Loreto, Ucayali, Huánuco, San Martín y Amazonas, todas en regiones amazónicas colindantes con Brasil; así también, como en otras de regiones andinas como Cajamarca (Jaén) y Ayacucho donde se han detectado pacientes no diagnosticados, ubicados por búsqueda de organismos no gubernamentales que colaboran en la eliminación de la lepra en el Perú.

La organización sanitaria para el control de la lepra en el Perú, después de una evolución histórica errática, está a cargo a nivel central, de una dependencia administrativa normativa denominada Estrategia Sanitaria Nacional de Prevención y Control de la Tuberculosis - Componente Lepra, dependiente de la Dirección General de Salud de las Personas, que no ofrece, por su composición y recursos limitados, confiabilidad; con el agravante de que la última "Norma Técnica de Salud para Eliminación de la Lepra" data del año 2005, la información exhibida data del año 2006 y la recogida recientemente contrasta
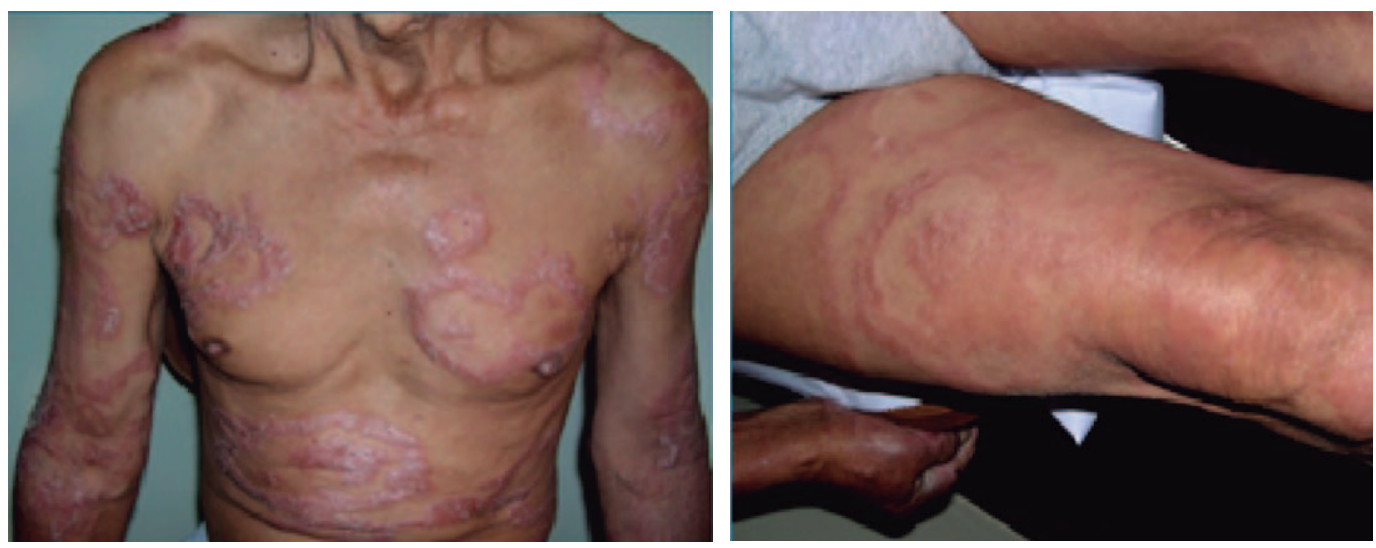

Figura 3.

Paciente con

lepra lepromatosa diagnosticado en Lima. Agricultor de 66 años de edad, procedente y residente en Huanta, Ayacucho, con 3 años de lesiones cutáneas no diagnosticadas, hasta presentar discapacidades motoras y atrofias 
con la realidad. Los organismos periféricos a cargo, por descentralización, del diagnóstico y control de la lepra en las zonas endémicas, no cuentan con el personal idóneo, ni realizan labores orientadas a la búsqueda activa de pacientes por no disponer de la capacidad ni recursos logísticos para cumplir con tal fin.

El Laboratorio Referencial Nacional de Diagnóstico de Lepra del INS/MINSA, que cumplía funciones de diagnóstico, investigación y capacitación de personal, se encuentra desactivado. Se están diagnosticando en la capital de la república casos de lepra lepromatosa altamente bacilífera, procedentes de las regiones endémicas, que no se han diagnosticado en su lugar de origen y que se encuentran en condiciones de convivencia y contacto de alto riesgo para la población circundante. Por lo que hay que concluir que el país se encuentra, todavía, en riesgo epidemiológico de producirse recrudescencia de la lepra en zonas endémicas y su propagación a otras que ofrecen condiciones de receptividad para esta peligrosa enfermedad. Los proyectos en ejecución de vías de comunicación interoceánicas entre el Perú y Brasil que discurrirán por áreas endémicas leprógenas ofrecen el peligro, que antaño se produjo, de una nueva propagación de la lepra, si no se toman las medidas sanitarias que permitan su control que actualmente se han relajado.

\section{REFERENCIAS BIBLIOGRÁFICAS}

1. Organización Mundial de la Salud. Guía para la eliminación de la lepra como problema de salud pública. Ginebra: OMS; 1995.

2. World Health Organization. Global leprosy situation, 2010. Wkly Epidemiol Rec. 2010 Aug 27;85(35):337-48.

3. Noordeen $S$. The epidemiology of leprosy. In: Hastings RC (ed). Leprosy. 2nd ed. Edinburgh: Churchill Livingstone; 1994. p. 68.

4. Durrheim DN, Spearre R. Global leprosy elimination: time to change more than elimination target date. J Epidemiol Community Health. 2003 May;57(5):316-7.

5. Lockwood DN. Leprosy elimination - a virtual phenomenon or a reality? BMJ. 2002 Jun 22;324(7352):1516-8.

6. Pérez M. La Lepra. La Enfermedad Olvidada ¿Acabaremos algún día con ella? Fontilles, Rev. leprol. 2001; 23:5-6.
7. Organización Mundial de la Salud. Quimioterapia de la lepra: informe de un grupo de estudio de la OMS. Serie de Informes Técnicos N. ${ }^{\circ} 847$. Ginebra: OMS; 1994.

8. Manrique-Chicoma H, Reyes-Puma N, Palacios-Agúero $\mathrm{O}$, Chunga-Ruiz $\mathrm{D}$, Laguna-Torres A, Huamán-Gonzales L. Conocimientos, actitudes y creencias del personal de salud acerca del mal de Hansen. Rev Per Enf Inf Trop. 2001;1(2):78-83.

9. Pesce H. La Epidemiologia de la Lepra en el Perú. Tesis Doctoral. Lima, Perú: Universidad Nacional Mayor de San Marcos; 1961.

10. Burstein Z. La lepra, enfermedad paradigmática, ¿podrá ser eliminada en un futuro cercano? Folia Dermatol 2002;13(2):51-6
11. Burstein Z. Quiebra del Programa de Control de la Lepra en el Perú por la descentralización e integración a los programas generales de salud. Arch Argent Dermat. 1980;30(3):173-80.

12. Perú, Ministerio de Salud. Norma Técnica de Salud para la Eliminación de la Lepra. N.T.S N ${ }^{\circ} 39$ MINSA/ DGSPV.01 2005. Lima: MINSA; 2005.

13. Neyra J. Evaluación Operacional y epidemiologia de la hanseniasis en el departamento de Ucayali 1980-1984. Bol Soc Per Enf Inf Trop. 1985;4:13-7.

Correspondencia: Zuño Burstein Dirección: Cápac Yupanqui 1400, Jesús María, Lima, Perú

Teléfono: (511) 471-9920 anexo: 122

Correoelectrónico:zburstein@ins.gob.pe 